\title{
NITROGEN ABUNDANCES IN GIANT STARS OF THE GLOBULAR CLUSTER NGC $6752^{1}$
}

\author{
DAVID YoNG \\ Research School of Astronomy and Astrophysics, Australian National University, Mount Stromlo Observatory, \\ Cotter Road, Weston Creek, ACT 2611, Australia; yong@mso.anu.edu.au \\ FRANK GRUNDAHL \\ Department of Physics and Astronomy, University of Aarhus, \\ DK-8000 Aarhus C, Denmark; fgj@phys.au.dk \\ JeNNIFER A. JoHnson \\ Department of Astronomy, Ohio State University, Columbus, OH 43210; \\ jaj@astronomy.ohio-state.edu \\ AND \\ Martin Asplund \\ Max-Planck-Institut für Astrophysik, Karl-Schwarzschild-Strasse 1, Postfach 1317, \\ 85741 Garching bei München, Germany; asplund@mpa-garching.mpg.de \\ Received 2008 April 16; accepted 2008 May 30
}

\begin{abstract}
We present $\mathrm{N}$ abundances for 21 bright giants in the globular cluster NGC 6752 based on high-resolution UVES spectra of the $3360 \AA \mathrm{NH}$ lines. We confirm that the Strömgren $c_{1}$ index traces the $\mathrm{N}$ abundance and find that the starto-star $\mathrm{N}$ abundance variation is $1.95 \mathrm{dex}$, at the sample's luminosity. We find statistically significant correlations, but small-amplitude variations, between the abundances of $\mathrm{N}$ and $\alpha$-, Fe-peak, and $s$-process elements. Analyses using model atmospheres with appropriate $\mathrm{N}, \mathrm{O}, \mathrm{Na}$, and $\mathrm{Al}$ abundances would strengthen, rather than mute, these correlations. If the small variations of heavy elements are real, then the synthesis of the $\mathrm{N}$ anomalies must take place in stars which also synthesize $\alpha$-, Fe-peak, and $s$-process elements. These correlations offer support for contributions from both asymptotic giant branch and massive stars to the globular cluster abundance anomalies.
\end{abstract}

Subject headings: Galaxy: abundances - globular clusters: individual (NGC 6752) - stars: abundances

Online material: color figures

\section{INTRODUCTION}

Several decades have passed since the first identifications of the star-to-star abundance variations of light elements in globular clusters (Popper 1947; Osborn 1971; Norris \& Freeman 1979). Hydrogen burning via the $\mathrm{CNO}, \mathrm{Ne}-\mathrm{Na}$, and $\mathrm{Mg}-\mathrm{Al}$ chains at high temperatures qualitatively accounts for the observed trends: the abundances of $\mathrm{C}$ and $\mathrm{O}$ are low when $\mathrm{N}$ is high; $\mathrm{O}$ and $\mathrm{Na}$ are anticorrelated, as are $\mathrm{Mg}$ and $\mathrm{Al}$ (Langer \& Hoffman 1995; Denissenkov et al. 1998; Karakas \& Lattanzio 2003). While the amplitude of the variation may differ from cluster to cluster, the abundance patterns have been found in every well-studied Galactic globular cluster (Smith 1987; Kraft 1994; Gratton et al. 2004). Such abundance patterns have also been identified in stars in extragalactic globular clusters (Letarte et al. 2006; Johnson et al. 2006). However, the origin of these abundance anomalies remains elusive.

Internal nucleosynthesis and mixing can account for $\mathrm{C}, \mathrm{N}$, and $\mathrm{Li}$ in giant stars whose abundances exhibit a dependence on evolutionary status (Suntzeff \& Smith 1991; Grundahl et al. 2002). These abundance patterns, $\mathrm{C}$ and $\mathrm{Li}$ destruction along with $\mathrm{N}$ production, are also found in field stars as they evolve up the red giant branch (RGB; Gratton et al. 2000). However, the identification of $\mathrm{C}, \mathrm{N}, \mathrm{O}, \mathrm{Na}, \mathrm{Mg}$, and $\mathrm{Al}$ abundance variations in unevolved cluster stars (Briley et al. 1996; Gratton et al. 2001;

\footnotetext{
${ }^{1}$ Based on observations made with ESO Telescopes at the Paranal Observatories under program 65.L-0165(A).
}

Grundahl et al. 2002; Cohen \& Meléndez 2005) demonstrates that an external pollution mechanism must be the dominant source, since unevolved stars have insufficient internal temperatures to run the necessary nuclear reactions and lack a mechanism to mix the products to surface layers. Unlike $\mathrm{C}, \mathrm{N}$, and $\mathrm{Li}$, abundance variations for the elements $\mathrm{O}-\mathrm{Al}$ have rarely, if ever, been identified in field stars, indicating that the abundance anomalies are likely associated with some (presently unknown) property of the cluster environment. Fulbright et al. (2007) found low O and high $\mathrm{Na}$ and $\mathrm{Al}$ abundances in two bulge giants. However, they suggest that these two stars may be members of the bulge globular cluster NGC 6522. Intermediate-mass asymptotic giant branch (AGB) stars (Cottrell \& Da Costa 1981; Ventura \& D'Antona 2005) and massive stars (Prantzos \& Charbonnel 2006; Smith 2006; Decressin et al. 2007) are candidates for the external pollution scenario. However, neither scenario currently provides a satisfactory explanation.

Nitrogen abundance variations in globular cluster giants were inferred from early observations of $\mathrm{CH}$ and $\mathrm{CN}$ variations (Zinn 1977; Da Costa \& Cottrell 1980). The NH molecular lines arguably offer the best probe of $\mathrm{N}$ abundances, in contrast to analyses of the $\mathrm{CN}$ lines, which require knowledge of the $\mathrm{C}$ and $\mathrm{O}$ abundances. However, few studies have measured $\mathrm{N}$ from $\mathrm{NH}$ as well as the abundances for additional elements in a large homogeneous sample. In particular, few studies have measured $\mathrm{N}$ from $\mathrm{NH}$ using high-resolution spectra for a large sample. In this paper, we perform such an analysis of bright giants in the globular cluster NGC 6752 to measure the amplitude of the $\mathrm{N}$ abundance variation and 


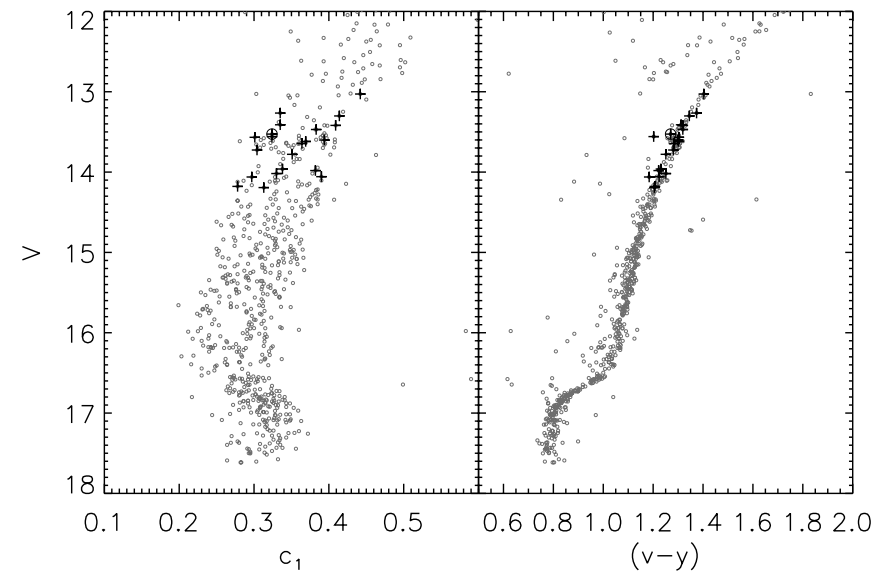

FIG. 1.-The $V$ vs. $c_{1}(l e f t)$ and $V$ vs. $(v-y)(r i g h t)$ color-magnitude diagrams using the Grundahl et al. (1999) photometry. The plus signs indicate the locations of our targets. (The large open circle marks NGC 6752-7.)

to identify correlations between $\mathrm{N}$ abundances and light-element abundances, as well as possible correlations between $\mathrm{N}$ abundances and the abundances of heavy elements.

\section{OBSERVATIONS AND DATA REDUCTION}

The targets were the same as those analyzed by Grundahl et al. (2002). The sample consists of 21 stars near the RGB bump, selected from the Strömgren $u v b y$ photometry of Grundahl et al. (1999) and observed in service mode in 2000 April-May using UVES (UV-Visual Echelle Spectrograph; D'Odorico et al. 2000). The stars were selected with the expectation that they would sample the full range of the star-to-star light-element abundance variation, at this luminosity. Grundahl et al. (2002) suspected that the Strömgren $c_{1}$ index was tracing the $\mathrm{N}$ abundance (the $u$ filter includes the $3360 \AA \mathrm{NH}$ features), and they identified clear correlations between $c_{1}$ and $\mathrm{NH}$ and $\mathrm{CN}$ indices defined by Briley
\& Smith (1993). The stars were deliberately selected to span the full range of the Strömgren $c_{1}$ index. In Figure 1 we show the $V$ versus $(v-y)$ and the $V$ versus $c_{1}$ color-magnitude diagrams showing the locations of the targets.

The ESO pipeline-reduced spectra have a resolution of $R=$ 60,000 , but were smoothed with a 5 pixel boxcar function to increase the signal-to-noise ratio $(\mathrm{S} / \mathrm{N})$. The resolving power after smoothing is $R \simeq 30,000$. Although the $\mathrm{S} / \mathrm{N}$ in the smoothed spectra is difficult to estimate in the crowded region near the $3360 \AA$ $\mathrm{NH}$ lines, we estimate that all stars have a typical $\mathrm{S} / \mathrm{N}=70$ per resolution element. For more details regarding target selection, observations, and data reduction, see Grundahl et al. (2002).

\section{STELLAR PARAMETERS AND ABUNDANCE ANALYSIS}

Derivation of the stellar parameters is described in Grundahl et al. (2002). Briefly, the effective temperatures $\left(T_{\text {eff }}\right)$ were determined from the Grundahl et al. (1999) uvby photometry using the Alonso et al. (1999) color-temperature relations. The surface gravities $(\log g)$ were estimated using the stellar luminosities and $T_{\text {eff }}$. The microturbulent velocities $\left(\xi_{t}\right)$ were derived in the usual way by forcing the abundances from $\mathrm{Fe}$ I to be independent of line strength. The stellar parameters for the program stars are presented in Table 1.

Visual examination of the spectra and the considerable range in NH indices measured by Grundahl et al. (2002) indicate a large amplitude to the star-to-star $\mathrm{N}$ abundance variation. Quantitative $\mathrm{N}$ abundances were derived by comparing the observed spectra with synthetic spectra generated using the LTE spectrum synthesis and line analysis package MOOG (Sneden 1973). The molecular data and subsequent line list for the $(0,0)$ and $(1,1)$ bands of the $A-X$ electronic transition of the NH molecule at $3360 \AA$ were taken from Johnson et al. (2007). We used model atmospheres computed by Kurucz (1993), which are the same as those used by Yong et al. $(2003,2005)$. Synthetic spectra were generated with

TABLE 1

Stellar Parameters and Nitrogen Abundances

\begin{tabular}{|c|c|c|c|c|c|c|c|c|c|}
\hline Name $1^{\mathrm{a}}$ & Name 2 & $\begin{array}{c}\text { R.A. } \\
(\mathrm{J} 2000.0)\end{array}$ & $\begin{array}{c}\text { Decl. } \\
(\mathrm{J} 2000.0)\end{array}$ & $V$ & $T_{\text {eff }}$ & $\log g$ & $\xi_{t}$ & {$[\mathrm{Fe} / \mathrm{H}]$} & {$[\mathrm{N} / \mathrm{Fe}]$} \\
\hline & NGC 6752-0 & 191103 & -595932 & 13.03 & 4699 & 1.83 & 1.47 & -1.62 & 1.37 \\
\hline B2882 ................... & NGC 6752-1 & 191047 & -600043 & 13.27 & 4749 & 1.95 & 1.41 & -1.58 & 0.27 \\
\hline B1635 ..................... & NGC 6752-2 & 191111 & $\begin{array}{llll}-60 & 00 & 17\end{array}$ & 13.30 & 4779 & 1.98 & 1.39 & -1.59 & 1.52 \\
\hline B2271 ..... & NGC 6752-3 & 191100 & -595640 & 13.41 & 4796 & 2.03 & 1.42 & -1.64 & 0.07 \\
\hline 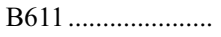 & NGC 6752-4 & 191133 & -600002 & 13.42 & 4806 & 2.04 & 1.40 & -1.61 & 1.37 \\
\hline B3490 ..................... & NGC 6752-6 & 191034 & -595955 & 13.47 & 4804 & 2.06 & 1.40 & -1.61 & 1.22 \\
\hline 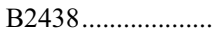 & NGC 6752-7 & 191057 & -600041 & 13.53 & 4829 & 2.10 & 1.33 & -1.84 & -0.33 \\
\hline B3103 ..................... & NGC 6752-8 & 191045 & -595818 & 13.56 & 4910 & 2.15 & 1.33 & -1.62 & 0.12 \\
\hline B3880 ..................... & NGC 6752-9 & 191026 & -595905 & 13.57 & 4824 & 2.11 & 1.38 & -1.63 & -0.03 \\
\hline B1330 ..................... & NGC $6752-10$ & 191118 & -595942 & 13.60 & 4836 & 2.13 & 1.37 & -1.60 & 1.27 \\
\hline B2728 .................... & NGC 6752-11 & 191050 & -600225 & 13.62 & 4829 & 2.13 & 1.32 & -1.64 & 1.07 \\
\hline B4216 ..................... & NGC 6752-12 & 191020 & -600030 & 13.64 & 4841 & 2.15 & 1.34 & -1.62 & 1.02 \\
\hline B2782 .................... & NGC $6752-15$ & 191049 & -600155 & 13.73 & 4850 & 2.19 & 1.35 & -1.61 & -0.43 \\
\hline B4446...... & NGC 6752-16 & 191015 & -595914 & 13.78 & 4906 & 2.24 & 1.32 & -1.60 & 1.32 \\
\hline \multirow[t]{3}{*}{ B1113 ….................. } & NGC 6752-19 & 191123 & -595940 & 13.96 & 4928 & 2.32 & 1.29 & -1.61 & 0.72 \\
\hline & NGC $6752-20$ & 191036 & -595608 & 13.98 & 4929 & 2.33 & 1.32 & -1.59 & 1.32 \\
\hline & NGC 6752-21 & 191113 & -600230 & 14.02 & 4904 & 2.33 & 1.29 & -1.61 & 0.87 \\
\hline \multirow[t]{4}{*}{ 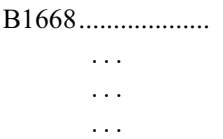 } & NGC 6752-23 & 191112 & -595829 & 14.06 & 4916 & 2.35 & 1.27 & -1.62 & 1.37 \\
\hline & NGC 6752-24 & 191044 & -595941 & 14.06 & 4948 & 2.37 & 1.15 & -1.65 & -0.18 \\
\hline & NGC 6752-29 & 191017 & -600100 & 14.18 & 4950 & 2.42 & 1.26 & -1.64 & -0.33 \\
\hline & NGC $6752-30$ & 191039 & -595947 & 14.19 & 4943 & 2.42 & 1.27 & -1.62 & 0.52 \\
\hline
\end{tabular}

NoтE.-Units of right ascension are hours, minutes, and seconds, and units of declination are degrees, arcminutes, and arcseconds.

a Star names from Buonanno et al. (1986). 


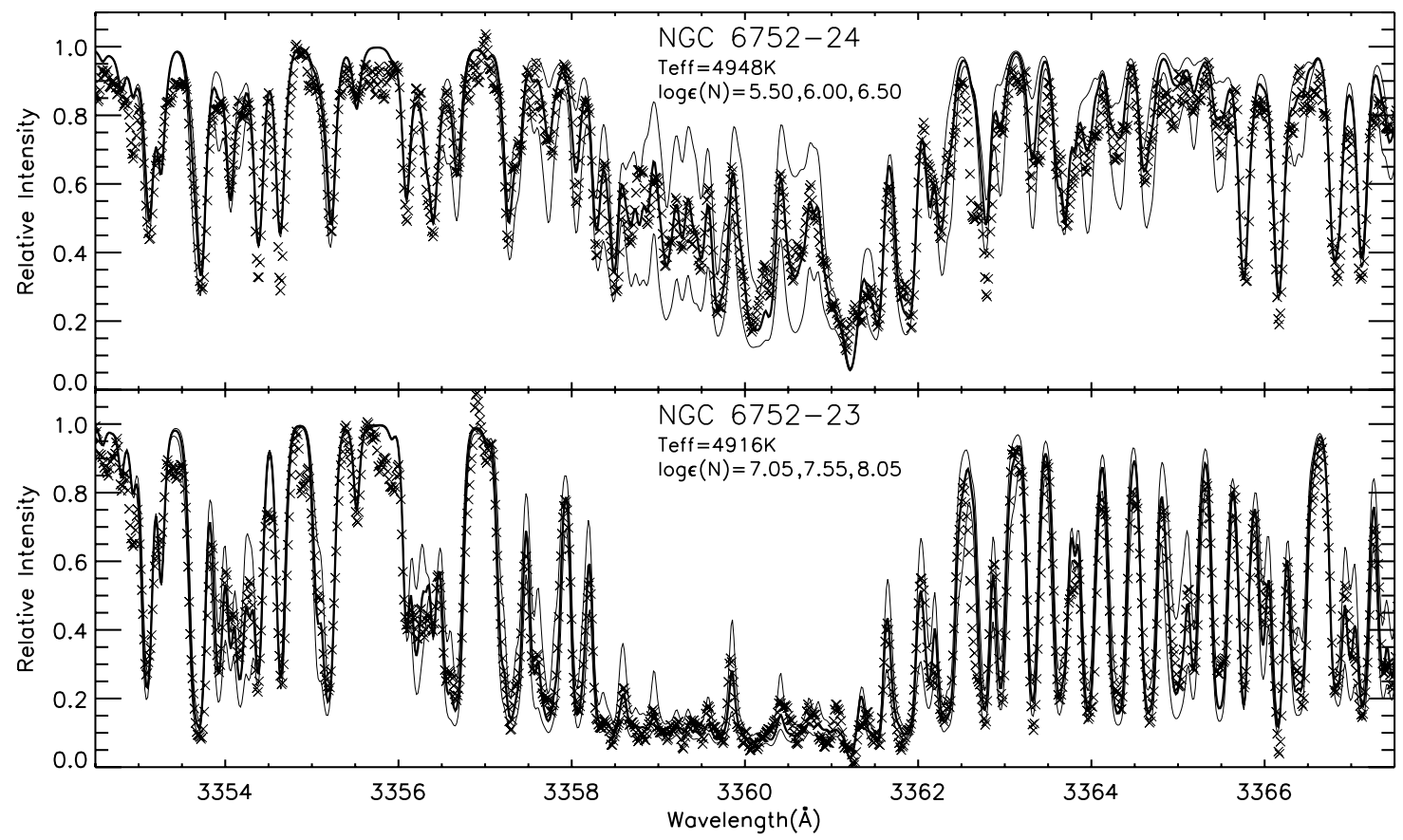

FIG. 2.-Observed spectra (crosses) for NGC 6752-24 (upper panel) and NGC 6752-23 (lower panel) near the 3360 Å NH band. These stars have very similar stellar parameters. Synthetic spectra with different $\mathrm{N}$ abundances are shown. The thick black line represents the best fit, and unsatisfactory fits ( \pm 0.5 dex) are shown as lower thin and upper thin lines. [See the electronic edition of the Journal for a color version of this figure.]

different $\mathrm{N}$ abundances (we assumed that all $\mathrm{N}$ was in the form ${ }^{14} \mathrm{~N}$ ). The difficult task of setting the continuum was achieved by selecting a handful of continuum windows and using the synthetic spectra as a guide. We adjusted the $\mathrm{N}$ abundances until the residuals between the synthetic and observed spectra were minimized. In Figures 2 and 3 we show pairs of stars with essentially identical stellar parameters. However, these pairs of stars have considerably different $\mathrm{N}$ abundances, as can be seen qualitatively by eye or quantitatively via the $c_{1}$ index and final [N/Fe] abundance. The $\mathrm{N}$ abundances, $[\mathrm{N} / \mathrm{Fe}]$, are presented in Table 1 assum- ing the newly determined solar abundance of $\log \epsilon(N)=7.78$ (Grevesse et al. 2007). Our conclusions would not be changed had we adopted a previous value for the solar $\mathrm{N}$ abundance, e.g., $\log \epsilon(N)=7.92$ from Grevesse \& Sauval (1998).

We estimate that the internal uncertainties in the measured $\mathrm{N}$ abundances are typically $0.2 \mathrm{dex}$, resulting from errors in the adopted stellar parameters and errors in determining the best fit. The internal errors would be slightly lower $(\sim 0.15 \mathrm{dex})$ for the most N-poor stars, and slightly higher $(\sim 0.25 \mathrm{dex})$ for the most $\mathrm{N}$-rich stars due to saturation concerns. For additional elements

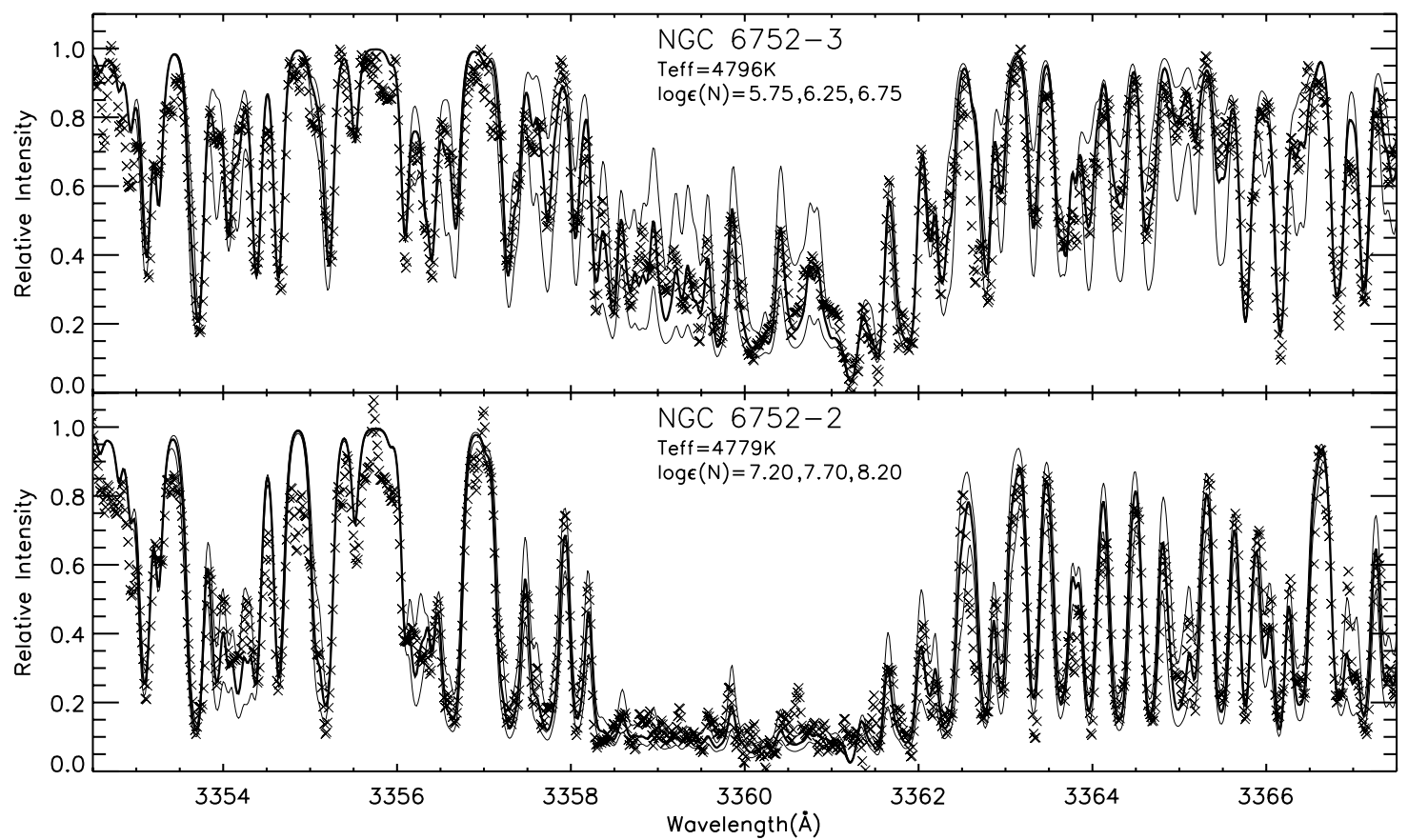

FIG. 3.-Same as Fig. 2, but for the slightly cooler pair of stars NGC 6752-3 (upper panel) and NGC 6752-2 (lower panel). [See the electronic edition of the Journal for a color version of this figure.] 


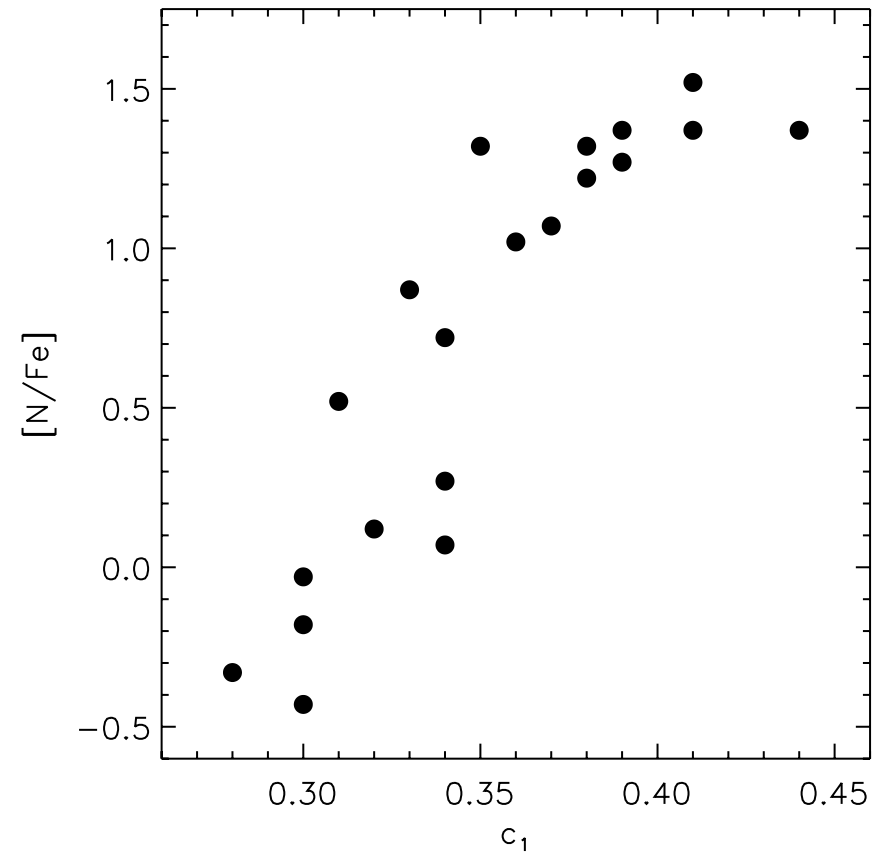

FIG. 4. - $[\mathrm{N} / \mathrm{Fe}]$ vs. $c_{1}$. Star NGC $6752-7$ is not included in this plot due to its deviating $[\mathrm{Fe} / \mathrm{H}]$.

discussed in $\S 4$, the derivation of abundances and their associated uncertainties were described in Grundahl et al. (2002) and Yong et al. (2003, 2005). ( We omit Star NGC 6752-7 from further analysis due to its deviating $[\mathrm{Fe} / \mathrm{H}]$.)

\section{DISCUSSION}

\subsection{Nitrogen Abundances and Comparisons with Previous Studies}

The $[\mathrm{N} / \mathrm{Fe}]$ ratios for the sample range from $[\mathrm{N} / \mathrm{Fe}]=-0.43$ to $[\mathrm{N} / \mathrm{Fe}]=+1.52$, almost a 2 dex variation. Recall that the sample was selected according to the Strömgren $c_{1}$ index, which Grundahl et al. (2002) suspected was tracing the $\mathrm{N}$ abundance. In Figure 4 we plot the $c_{1}$ index versus the $\mathrm{N}$ abundance. The clear correlation confirms that $c_{1}$ indeed traces the $\mathrm{N}$ abundance. Given the Strömgren $c_{1}$ selection criteria, it is not surprising that the full abundance distribution is well sampled. However, our $\mathrm{N}$ abundances are not representative of the $\mathrm{N}$ distribution for the entire cluster.

Carretta et al. (2005) measured $\mathrm{N}$ abundances for nine dwarfs and nine subgiants in NGC 6752. Their N measurements are based on analysis of the $\mathrm{CN}$ molecular lines (rather than the $\mathrm{NH}$ lines used in this analysis), which requires knowledge of the $\mathrm{C}$ and $\mathrm{O}$ abundances. Our syntheses of the NH lines, and therefore our $\mathrm{N}$ abundances, are entirely independent of the adopted $\mathrm{C}$ and $\mathrm{O}$ abundances, and presumably the uncertainties in our $\mathrm{N}$ abundances will be lower than analyses based on $\mathrm{CN}$ features. Aside from one subgiant with the abundance ratio $[\mathrm{N} / \mathrm{Fe}]=0.0$, the Carretta et al. (2005) sample spans the range $+1.0 \leq[\mathrm{N} / \mathrm{Fe}] \leq$ +1.7 . Their maximum abundance $[\mathrm{N} / \mathrm{Fe}]=+1.7$ is comparable to the maximum abundance derived in this study, within the measurement uncertainties. However, their lowest abundance $[\mathrm{N} / \mathrm{Fe}]=0.0$ is considerably higher than the minimum value found in our analysis. If $\mathrm{CN}$ cycling has occurred in our stars, which are more evolved than the Carretta et al. (2005) sample, we would expect our stars to have systematically higher $\mathrm{N}$ abundances (assuming that the two samples were drawn from the same

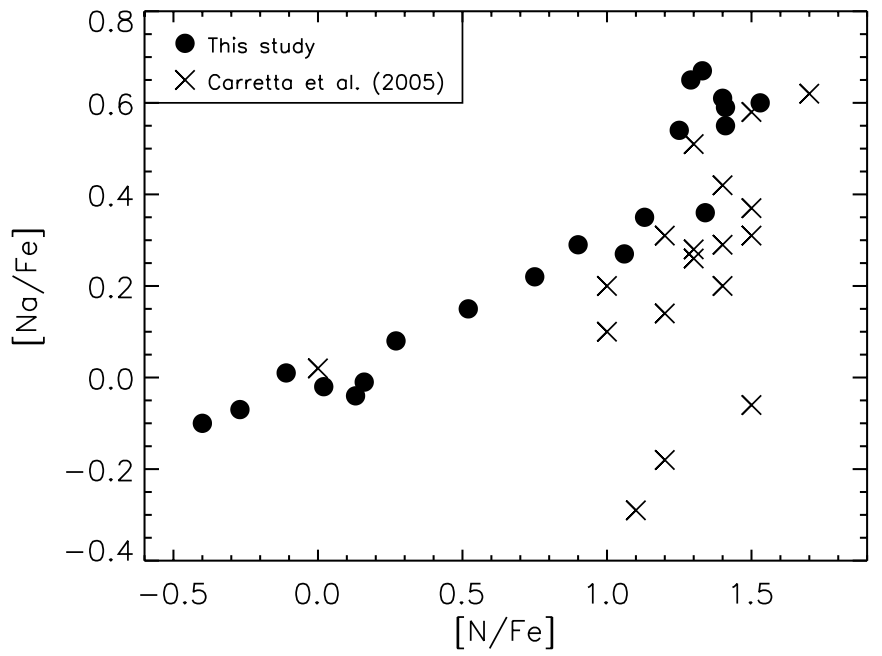

FIG. 5.- $[\mathrm{Na} / \mathrm{Fe}]$ vs. $[\mathrm{N} / \mathrm{Fe}]$ for this study (circles) and the Carretta et al. (2005) sample (crosses).

population and that the two samples covered the full range of the abundance variation at their respective luminosities).

For the Carretta et al. (2005) sample, abundances for other light elements known to vary from star to star have been derived by Gratton et al. (2001). For convenience, we consider the $[\mathrm{Na} / \mathrm{Fe}]$ ratio. In Figure 5 we compare $[\mathrm{Na} / \mathrm{Fe}]$ versus $[\mathrm{N} / \mathrm{Fe}]$ for our stars and the Carretta et al. (2005) stars. Their minimum value, $[\mathrm{Na} / \mathrm{Fe}]=-0.29$, is slightly lower than our minimum, $[\mathrm{Na} / \mathrm{Fe}]=$ -0.10 . The maximum $[\mathrm{Na} / \mathrm{Fe}]$ ratio for Carretta et al. (2005), $[\mathrm{Na} / \mathrm{Fe}]=+0.62$, is comparable to our maximum, $[\mathrm{Na} / \mathrm{Fe}]=$ +0.67 . Given that the amplitude of the $[\mathrm{Na} / \mathrm{Fe}]$ variation in Carretta et al. (2005) exceeds our [ $\mathrm{Na} / \mathrm{Fe}]$ variation, we would naively expect the $[\mathrm{N} / \mathrm{Fe}]$ variation in their sample to be comparable to, or larger than, our sample. Therefore, it is somewhat unusual that the amplitude of our $[\mathrm{N} / \mathrm{Fe}]$ variation is considerably larger than in Carretta et al. (2005). Inspection of Figure 5 shows that the $[\mathrm{N} / \mathrm{Fe}]$ distributions look rather different, and we speculate that the difference is due in part to difficulties in deriving accurate $\mathrm{N}$ abundances from $\mathrm{CN}$ lines. Although the Carretta et al. (2005) star with $[\mathrm{N} / \mathrm{Fe}]=0$ appears rather unusual compared to the bulk of their sample, it lies on the distribution defined by our stars. One possibility is that measurement uncertainties for $\mathrm{N}$ and/or Na have affected one or both of the analyses. We are not aware of additional analyses of $\mathrm{N}$ in large numbers of stars in NGC 6752.

Of the well-studied globular clusters, NGC 6752 exhibits one of the largest amplitudes for light-element abundance variations. For other well-studied globular clusters that also display large abundance variations, several have measured $\mathrm{N}$ abundances. Work by Briley et al. $(2002,2004)$ and Cohen et al. $(2002,2005)$ has shown that the clusters M5, M13, M15, M71, and 47 Tuc have $[\mathrm{N} / \mathrm{Fe}]$ ratios that cover 2 dex or more. These works are based on very large numbers of main-sequence stars $(\sim 50-100)$, albeit using lower resolution spectra with abundances determined via the comparison of indices measured in observed and synthetic spectra. These indices measure the flux removed by molecular features relative to nearby continuum bandpasses (Briley \& Cohen 2001). Carretta et al. (2005) also measured $\mathrm{N}$ abundances in modest numbers of dwarfs and subgiants in the globular clusters NGC 6397 and 47 Tuc. For NGC 6397, their analysis of subgiant stars shows that $[\mathrm{N} / \mathrm{Fe}]$ covers a range of $2.0 \mathrm{dex}$. For 47 Tuc, their analysis of dwarfs and subgiants shows a $[\mathrm{N} / \mathrm{Fe}]$ range of $1.6 \mathrm{dex}$. In summary, the amplitude of the $\mathrm{N}$ abundance variation in this 


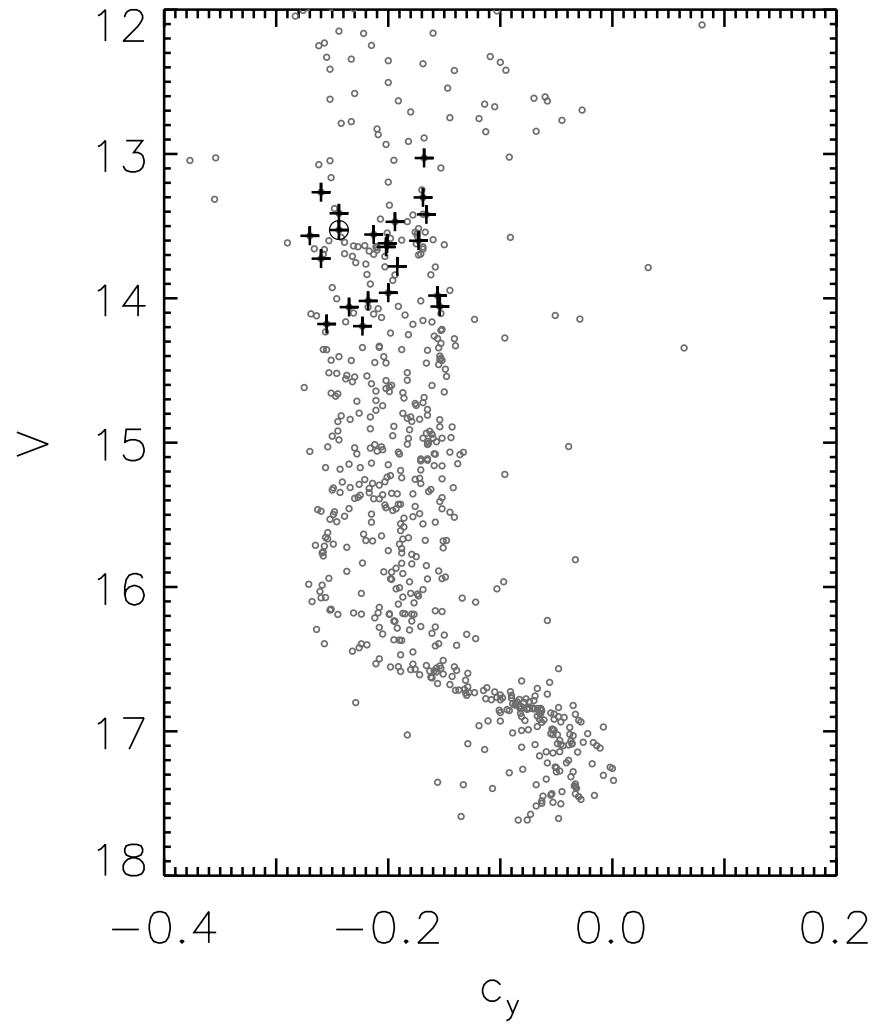

Fig. 6.-The $V$ vs. $c y$ color-magnitude diagram using the Grundahl et al. (1999) photometry. The plus signs indicate the locations of our targets. (The large open circle marks NGC 6752-7.)

study of NGC 6752 is comparable to that observed in other clusters.

\subsection{Strömgren Photometry and N Abundances}

To further investigate the relationship between Strömgren photometry and $\mathrm{N}$ abundances, we introduce a new index $c y=c_{1}-$ $(b-y)$. This index was identified through simple experiments, and is a purely empirical index which appears to remove temperature from $c_{1}$, to first order. The $c y$ index represents $c_{1}$ well, but for stars on the lower RGB (fainter than the bump), $c y$ appears to show no evolutionary component. In Figure 6 we plot $V$ versus $c y$. While there appears to be a large scatter in $c y$ at all evolutionary phases, the width and center of the distribution are independent of the $V$ magnitude. In Figure 7 we plot [N/Fe] versus $c y$. Remarkably, there appears to be a linear relation to which we fit $[\mathrm{N} / \mathrm{Fe}]=$ $16.11 c y+4.07$. The scatter around the fit is only 0.29 dex. A linear fit between $[\mathrm{N} / \mathrm{Fe}]$ and $c_{1}$ has a scatter of $0.31 \mathrm{dex}$, and a quadratic fit has a scatter of $0.27 \mathrm{dex}$. The reduced scatter for the quadratic fit confirms the impression from Figure 4. However, we prefer the linear relation between $[\mathrm{N} / \mathrm{Fe}]$ and $c y$, which can then be used to explore in more detail the distribution of $[\mathrm{N} / \mathrm{Fe}]$ for the large numbers of giant stars for which Strömgren photometry, but not high-resolution spectra, are available.

In Figure 8 we plot the $[\mathrm{N} / \mathrm{Fe}]$ distribution for all giant stars $(N=110)$ with $V$ magnitudes spanned by the calibration stars employing the relation given above. We find that the distribution increases toward higher $[\mathrm{N} / \mathrm{Fe}]$ values. That is, the distribution is not flat, nor is it bimodal. Norris et al. (1981) found that the CN distribution for NGC 6752 is bimodal based on 69 giant stars. In the same figure, we also plot the $[\mathrm{N} / \mathrm{Fe}]$ distribution for all giant stars with magnitudes $13 \leq V \leq 16(N=559)$. Although it is not clear whether the above relation is applicable beyond the mag-

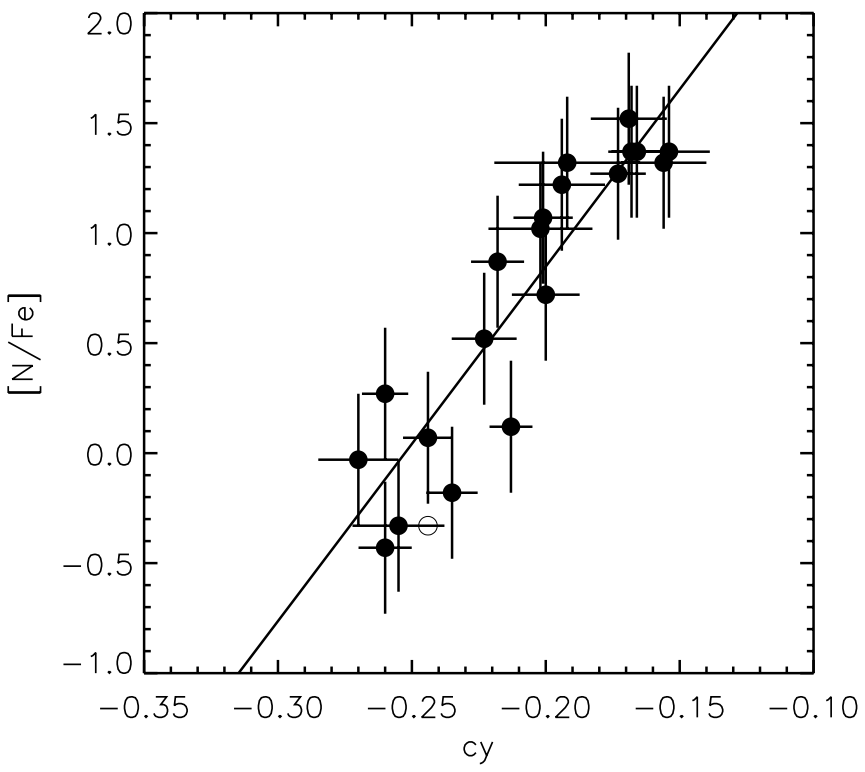

FIG. 7. - $[\mathrm{N} / \mathrm{Fe}]$ vs. $c y$. The straight line is the best fit to the data. (The large open circle marks NGC 6752-7.)

nitudes (i.e., stellar parameters) of the calibrating stars, we note that both distributions are very similar. These results imply that in other clusters, well-calibrated $u v b y$ photometry could be used to probe the nitrogen abundance distribution. This may even be possible for clusters in the LMC and SMC.

The $c y-[\mathrm{N} / \mathrm{Fe}]$ relation is valid over the range of parameters covered by our sample. Having applied this relation to the entire $\mathrm{RGB}$, we recover a similar $[\mathrm{N} / \mathrm{Fe}]$ distribution, as seen within the stars spanned by the calibrating stars. Therefore, we speculate that this relation may be applicable along the entire RGB.

\subsection{Correlations between the Abundances of Nitrogen and Other Elements}

Statistically significant correlations, albeit of small amplitude, between $\mathrm{Al}$ and the abundances of $\mathrm{Si}$ and heavier elements in NGC 6752's bright giant stars (including the RGB bump stars in this analysis) were identified by Yong et al. (2005). Since the $\mathrm{N}$ abundances exhibit a larger amplitude than the $\mathrm{Al}$ abundances, we seek to investigate whether there are correlations between $\mathrm{N}$ and heavier elements. In Figures 9-13 we plot the $\mathrm{N}$ abundances versus all measured elements in NGC 6752. In these figures, the abundances for elements other than $\mathrm{N}$ are taken from Grundahl et al. (2002) and Yong et al. (2003, 2005). All studies are homogeneous, using the same model atmospheres, stellar parameters, and analysis techniques.

For $\mathrm{O}, \mathrm{Na}, \mathrm{Mg}$, and $\mathrm{Al}$, the usual correlations with $\mathrm{N}$ are evident. For completeness, we note that these correlations are significant at the $10 \sigma$ level (i.e., we fit a straight line to the data and measure the slope and uncertainty, taking into account both the $x$ - and $y$-errors). We find that the elements $\mathrm{Si}, \mathrm{Ca}, \mathrm{Sc}, \mathrm{Ti}, \mathrm{Ni}, \mathrm{Cu}$, $\mathrm{Y}, \mathrm{Zr}, \mathrm{Ba}$, and $\mathrm{Nd}$ all show correlations with $\mathrm{N}$. Although the amplitude of the abundance variations for these heavier elements is small $(<0.2 \mathrm{dex})$, the correlations are statistically significant at the level $2 \sigma$ or greater. (Even if we increase the $x$ - and $y$-errors by $50 \%, \mathrm{Si}, \mathrm{Sc}, \mathrm{Cu}, \mathrm{Y}, \mathrm{Zr}$, and Ba remain correlated with $\mathrm{N}$ at the level $2 \sigma$ or greater.) Taken at face value, these correlations indicate that the nucleosynthetic source of the $\mathrm{N}$ abundance variation must also synthesize small amounts of these heavier elements. In Yong et al. (2005) we showed that the abundances are not 

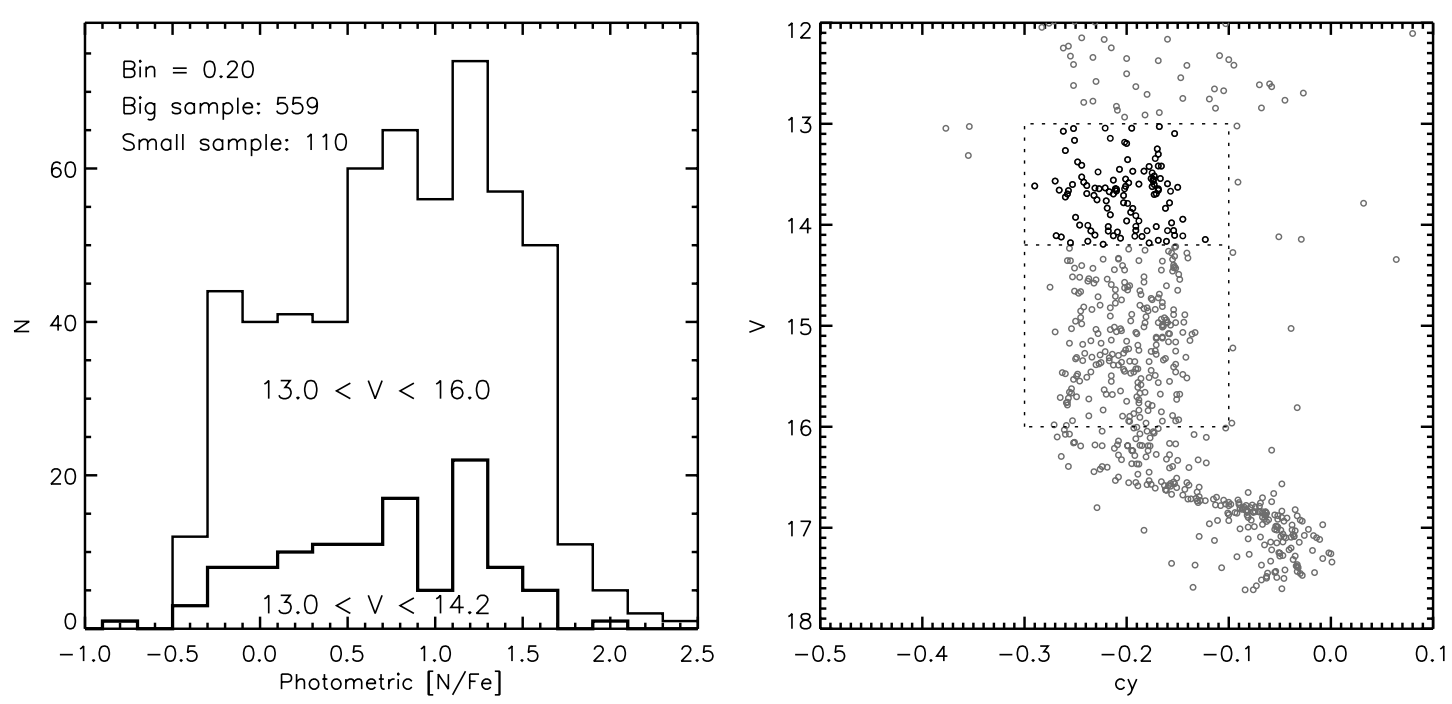

Fig. 8. - Left: $[\mathrm{N} / \mathrm{Fe}]$ distribution using the relation between $[\mathrm{N} / \mathrm{Fe}]$ and $c y$. The larger histogram shows all stars with $13 \leq V \leq 16$, while the smaller histogram shows only stars spanned by the calibrating stars. Both sets of stars are identified in the $V$ vs. $c y$ color-magnitude diagram in the right panel.

correlated with $T_{\text {eff }}$, and therefore any correlation between $\mathrm{N}$ and heavier elements is unlikely to be the result of systematic errors in the stellar parameters.

The model atmospheres used in the analysis were computed using scaled-solar compositions, whereas the stars to which these atmospheres were applied do not have scaled-solar compositions. Therefore, we were concerned that the correlations between $\mathrm{N}$ and heavier elements could be due to the use of inappropriate models. That is, would the derived abundances change if we used model atmospheres with the appropriately small or large $\mathrm{N}, \mathrm{O}, \mathrm{Na}$, and $\mathrm{Al}$ abundances? Or equivalently, does the inclusion of appropriate $\mathrm{N}, \mathrm{O}, \mathrm{Na}$, and $\mathrm{Al}$ abundances change the structure of the model atmospheres to such a degree that the derived abundances of Si and heavier elements are affected? Only small changes in the heavy-element abundances would be required to remove any correlations with $\mathrm{N}$.

In Table 2 we show the abundances for a subset of lines derived using four different model atmospheres. The model atmospheres
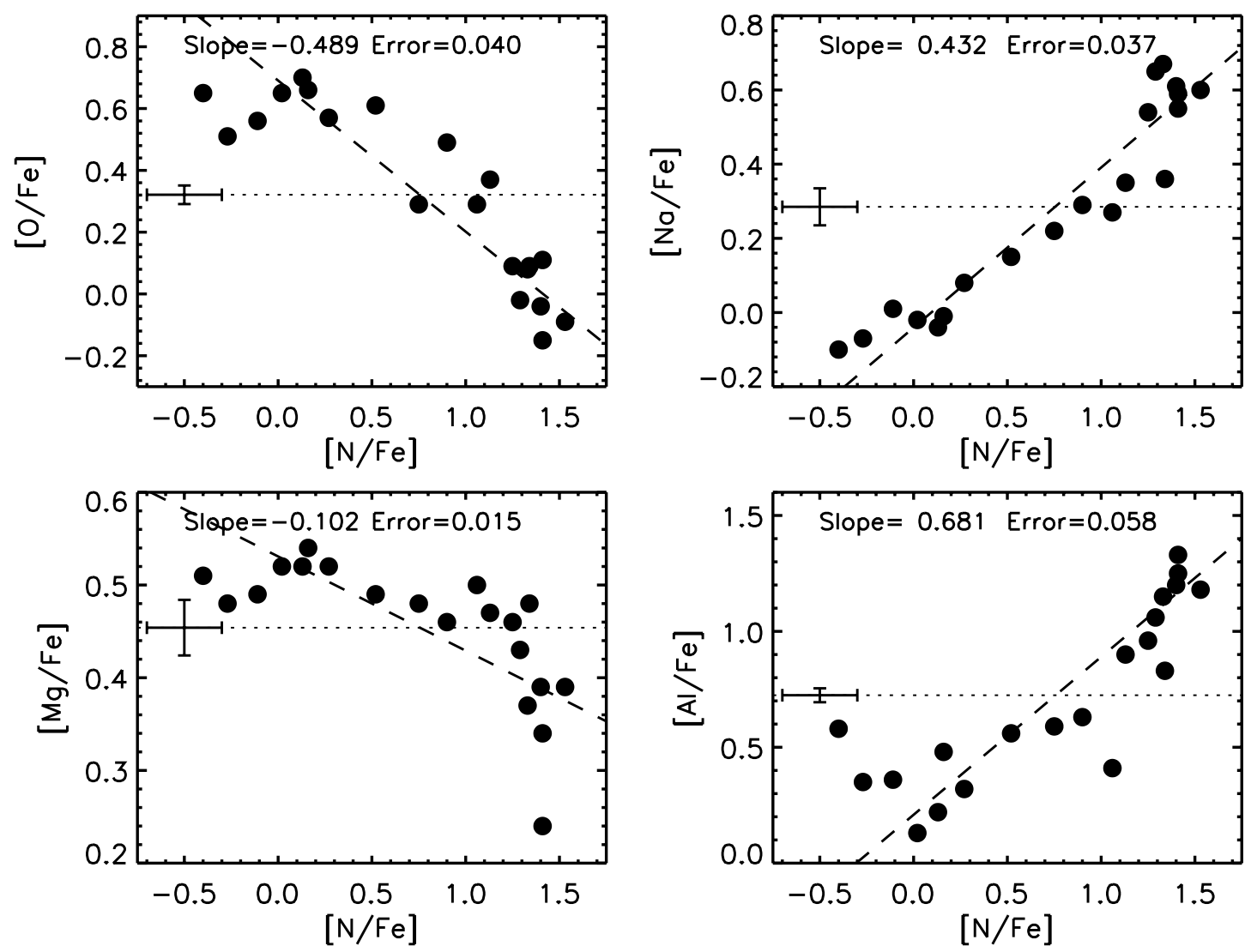

FIG. 9.- $[\mathrm{X} / \mathrm{Fe}]$ vs. $[\mathrm{N} / \mathrm{Fe}]$ for $\mathrm{O}, \mathrm{Na}, \mathrm{Mg}$, and Al. The error bar shows the $1 \sigma$ errors (see text for details). The dotted line is the mean abundance, and the dashed line is the linear least-squares fit to the data (slope and associated error are included). [See the electronic edition of the Journal for a color version of this figure.] 

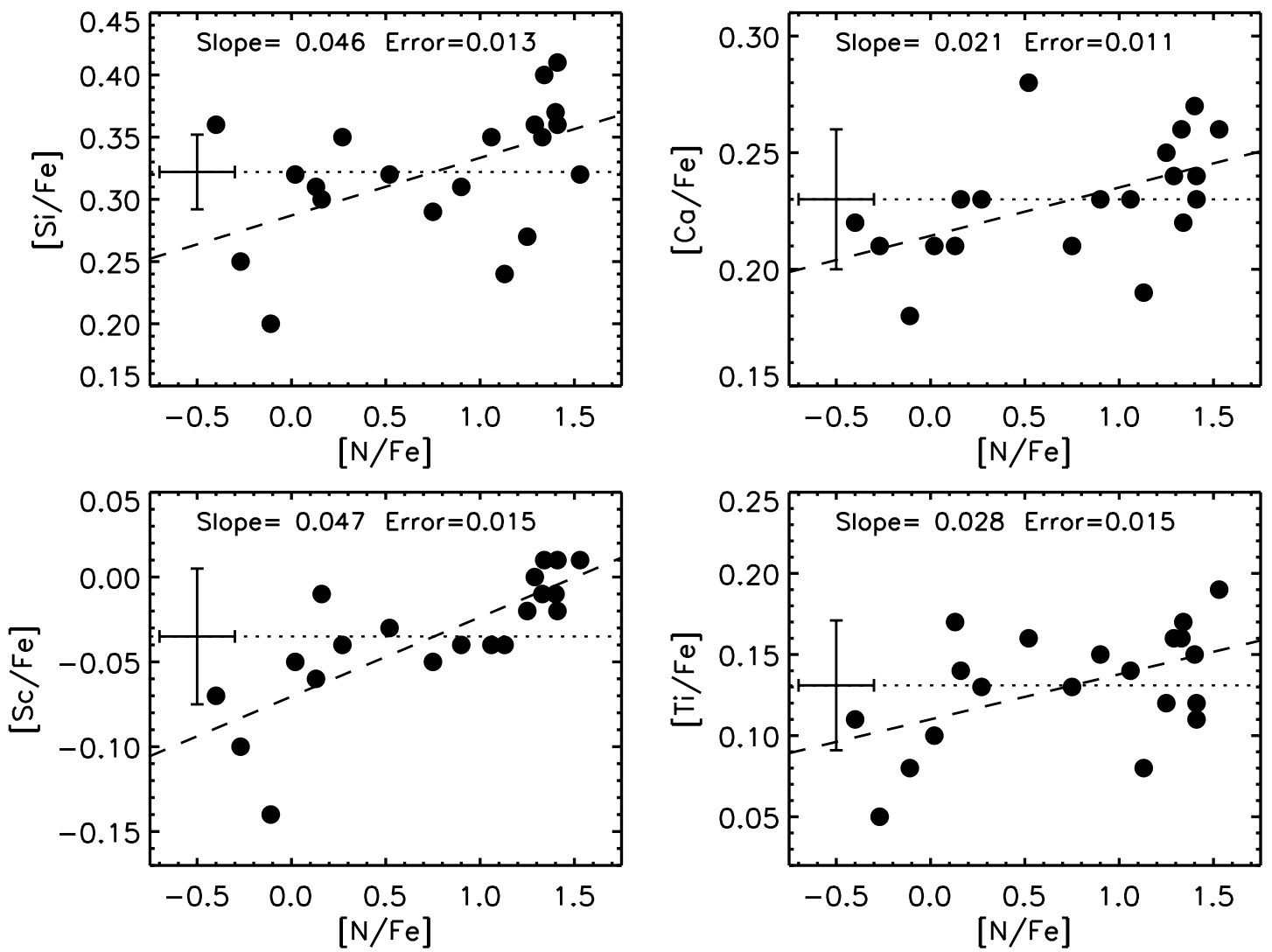

FIG. 10.- Same as Fig. 9, but for Si, Ca, Sc, and Ti. [See the electronic edition of the Journal for a color version of this figure.]
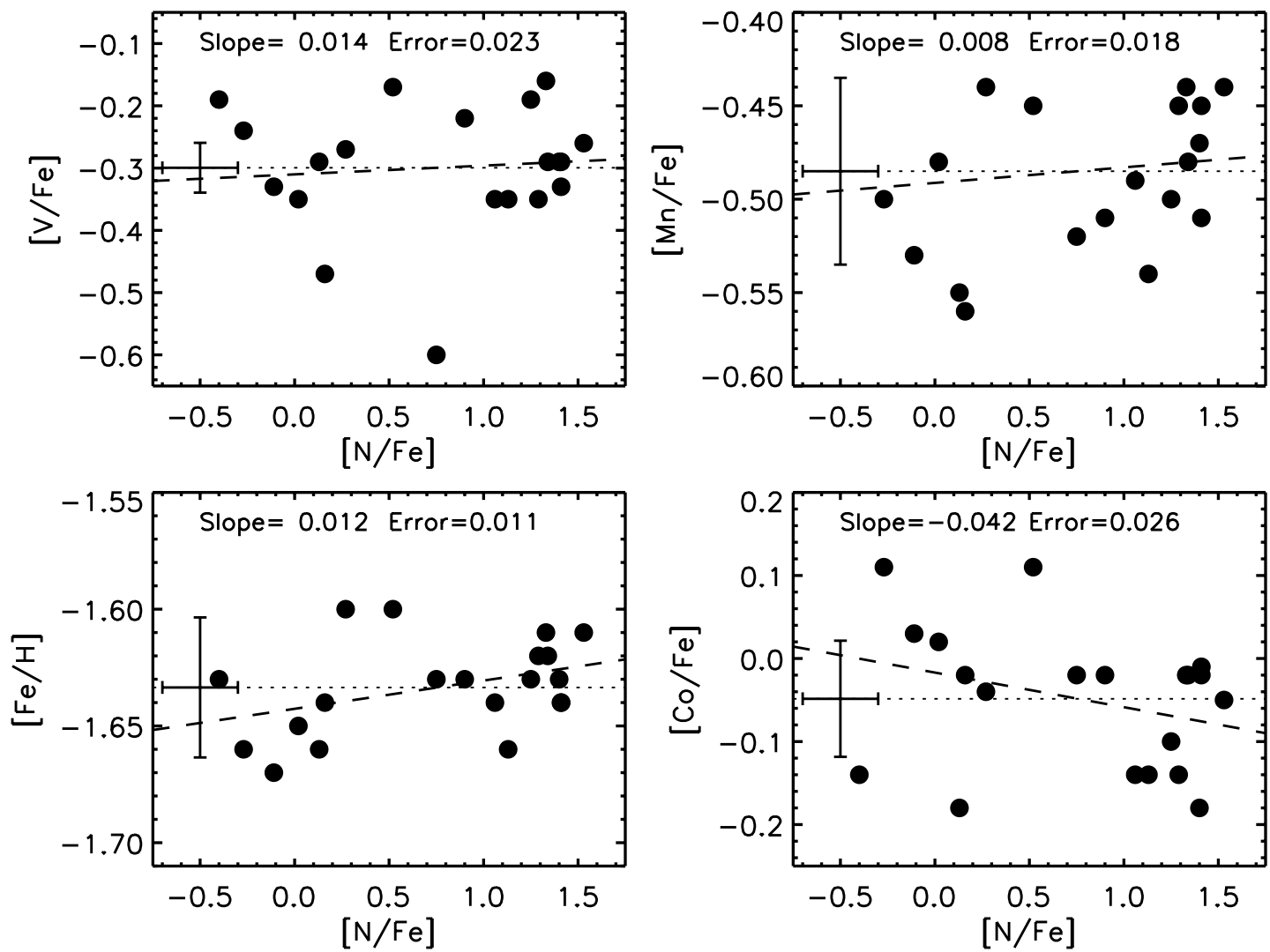

FIG. 11.- Same as Fig. 9, but for V, Mn, Fe, and Co. [See the electronic edition of the Journal for a color version of this figure.] 

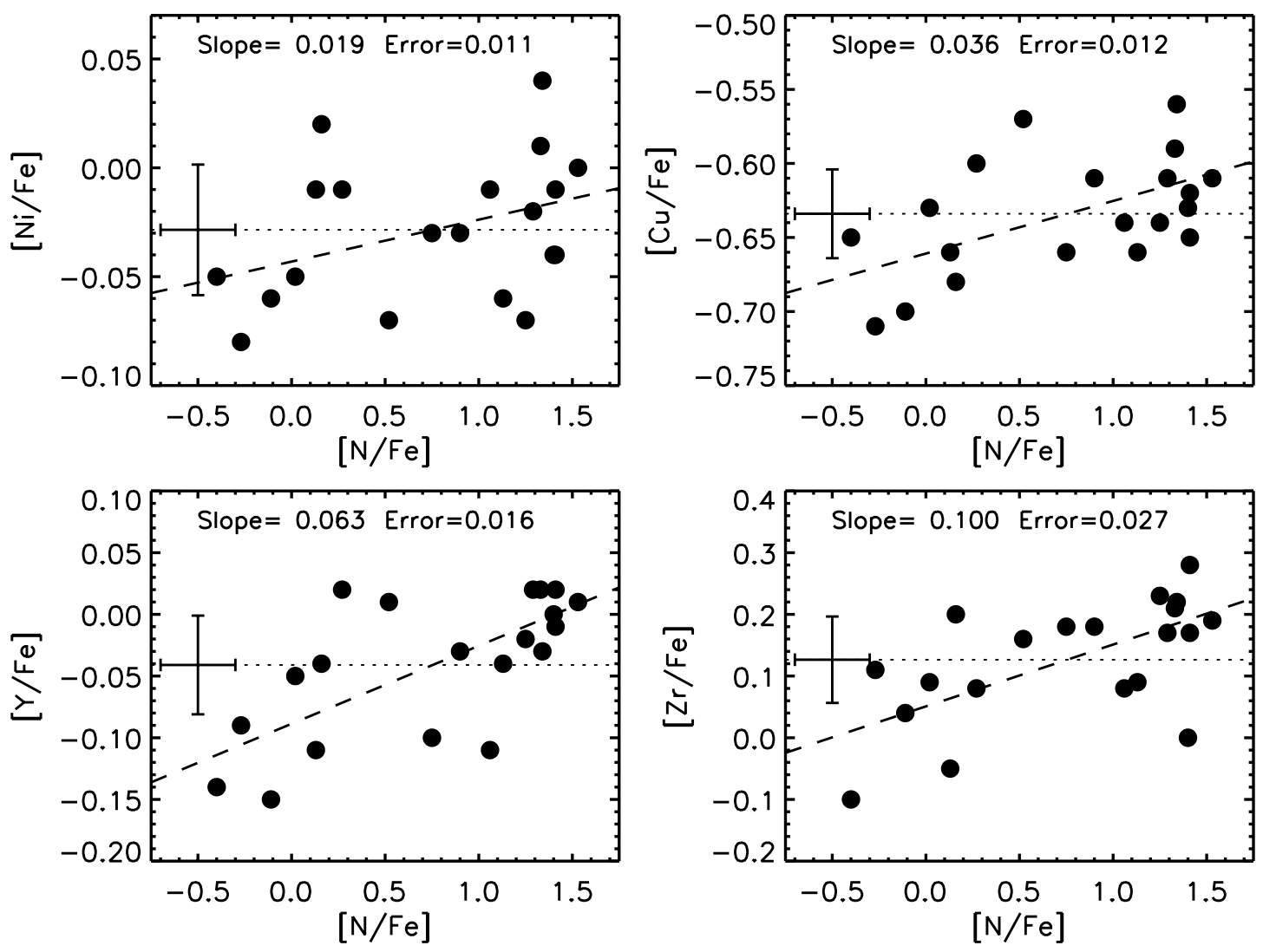

FIG. 12.-Same as Fig. 9, but for $\mathrm{Ni}, \mathrm{Cu}, \mathrm{Y}$, and $\mathrm{Zr}$. [See the electronic edition of the Journal for a color version of this figure.]
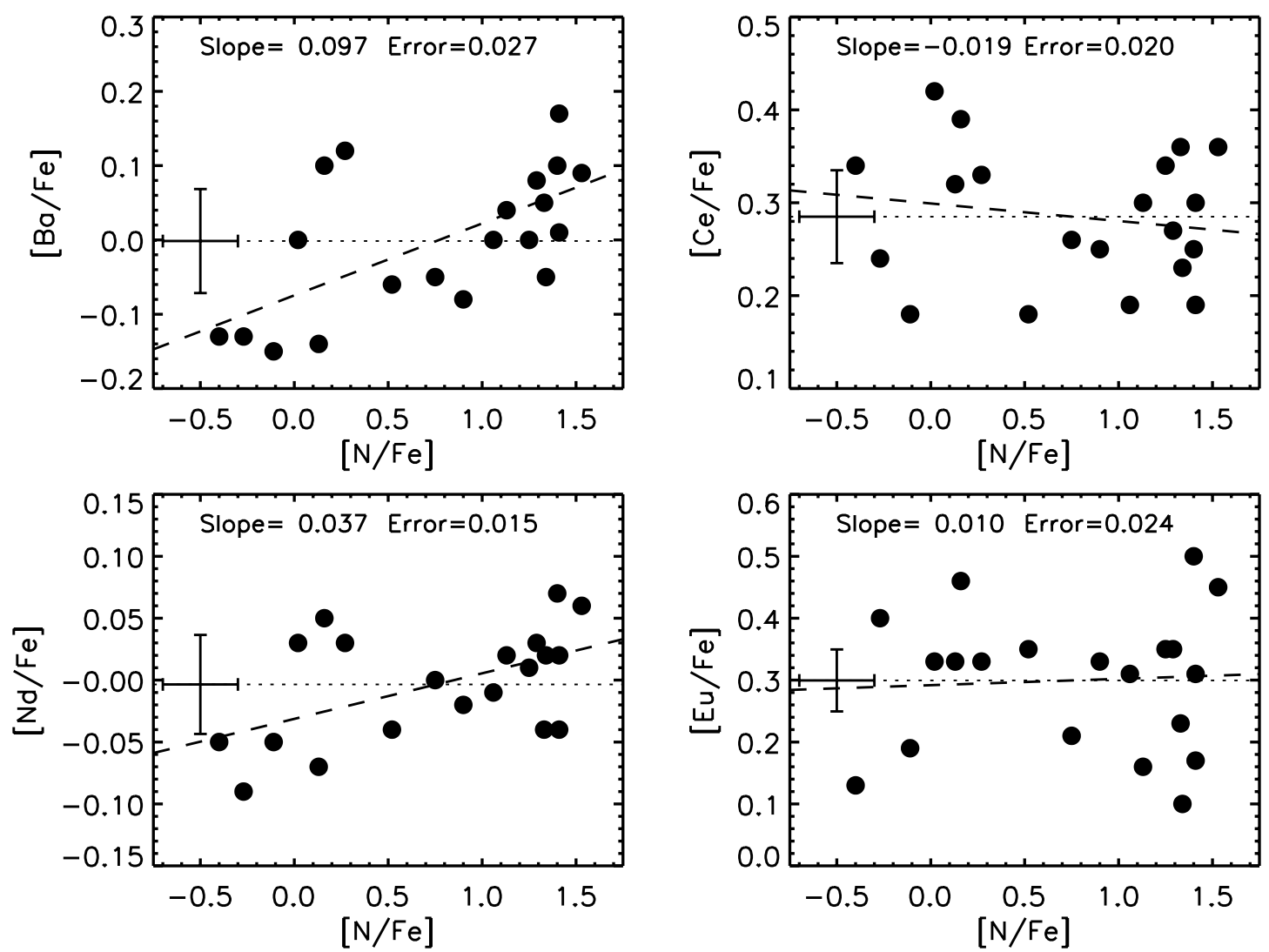

Fig. 13.-Same as Fig. 9, but for Ba, Ce, Nd, and Eu. [See the electronic edition of the Journal for a color version of this figure.] 
TABLE 2

Derived Abundances for Model Atmospheres with Scaled-Solar and Nonscaled-Solar Compositions

\begin{tabular}{|c|c|c|c|c|c|c|c|c|}
\hline \multirow[b]{2}{*}{ WAVELENGTH } & \multirow[b]{2}{*}{ SPECIES } & \multirow[b]{2}{*}{$\mathrm{eV}$} & \multirow[b]{2}{*}{$\log g f$} & \multirow[b]{2}{*}{ EW } & \multicolumn{4}{|c|}{ ABUNDANCE: $\log \epsilon(X)$} \\
\hline & & & & & Model $1^{\mathrm{a}}$ & Model $2^{\mathrm{b}}$ & Model $3^{\mathrm{c}}$ & Model $4^{\mathrm{d}}$ \\
\hline $5690.43 \ldots \ldots \ldots \ldots \ldots . .$. & 14.0 & 4.93 & -1.83 & 10.0 & 5.98 & 6.00 & 6.02 & 6.02 \\
\hline $6270.22 \ldots \ldots \ldots \ldots \ldots$ & 26.0 & 2.86 & -2.51 & 10.0 & 5.33 & 5.32 & 5.40 & 5.38 \\
\hline 6271.28 .................... & 26.0 & 3.33 & -2.76 & 10.0 & 6.13 & 6.13 & 6.20 & 6.18 \\
\hline 6416.92 ..................... & 26.1 & 3.89 & -2.74 & 10.0 & 5.64 & 5.70 & 5.70 & 5.75 \\
\hline $5509.91 \ldots \ldots \ldots \ldots \ldots . . .$. & 39.1 & 0.99 & -1.01 & 10.0 & 0.09 & 0.18 & 0.19 & 0.25 \\
\hline $6134.55 \ldots \ldots \ldots \ldots \ldots \ldots$ & 40.0 & 0.00 & -1.28 & 10.0 & 2.12 & 2.10 & 2.23 & 2.18 \\
\hline
\end{tabular}

a $T_{\text {eff }}=4780 \mathrm{~K}, \log g=2.0,[\mathrm{Fe} / \mathrm{H}]=-1.60$, scaled solar compositions.

${ }^{b}$ Same as model 1, but with $[\mathrm{N} / \mathrm{Fe}]=+1.5,[\mathrm{O} / \mathrm{Fe}]=-0.1,[\mathrm{Na} / \mathrm{Fe}]=+0.6,[\mathrm{Mg} / \mathrm{Fe}]=+0.4$, and $[\mathrm{Al} / \mathrm{Fe}]=+1.2$.

c $T_{\text {eff }}=4850 \mathrm{~K}, \log g=2.2,[\mathrm{Fe} / \mathrm{H}]=-1.60$, scaled solar compositions.

${ }^{d}$ Same as model 3, but with $[\mathrm{N} / \mathrm{Fe}]=-0.4,[\mathrm{O} / \mathrm{Fe}]=+0.6,[\mathrm{Na} / \mathrm{Fe}]=-0.1,[\mathrm{Mg} / \mathrm{Fe}]=+0.5$, and $[\mathrm{Al} / \mathrm{Fe}]=+0.6$.

and subsequent abundances were computed using the MARCS suite of software (Gustafsson et al. 1975; Asplund et al. 1997). Models 1 and 2 have identical $T_{\text {eff }}, \log g$, and [Fe/H]. However, model 1 has a scaled-solar composition, whereas model 2 has $[\mathrm{N} / \mathrm{Fe}]=+1.5,[\mathrm{O} / \mathrm{Fe}]=-0.1,[\mathrm{Na} / \mathrm{Fe}]=+0.6,[\mathrm{Mg} / \mathrm{Fe}]=$ +0.4 , and $[\mathrm{Al} / \mathrm{Fe}]=+1.2$, values which are essentially identical to those measured in the N-rich star NGC 6752-2. Similarly, models 3 and 4 have identical $T_{\text {eff }}, \log g$, and $[\mathrm{Fe} / \mathrm{H}]$. However, model 3 has a scaled-solar composition, whereas model 4 has $[\mathrm{N} / \mathrm{Fe}]=-0.4,[\mathrm{O} / \mathrm{Fe}]=+0.6,[\mathrm{Na} / \mathrm{Fe}]=-0.1,[\mathrm{Mg} / \mathrm{Fe}]=$ +0.5 , and $[\mathrm{Al} / \mathrm{Fe}]=+0.6$, values which are essentially identical to those measured in the N-poor star NGC 6752-15. For the purposes of these calculations, we assumed a strength of $10 \mathrm{~m} \AA$ to ensure that the lines are weak and on the linear part of the curve of growth. A comparison between the abundances derived using models 1 and 2 gauges the uncertainty when analyzing N-rich, Na-rich, Al-rich, O-poor, and Mg-poor stars. A comparison between the abundances derived using models 3 and 4 gauges the uncertainty for N-poor, Na-poor, Al-poor, O-rich, and Mg-rich stars. And a comparison between the abundances derived using models 2 and 4 gauges the uncertainty across the full abundance range. Given the small range in $T_{\text {eff }}(\sim 250 \mathrm{~K})$, we assume that the calculations would be applicable to all stars in our sample.

While the elements considered in Table 2 were chosen somewhat arbitrarily, they are neutral, and ionized species for which the correlations with $\mathrm{N}$ are significant ( $4 \sigma$ for $\mathrm{Y}$ and $3 \sigma$ for both $\mathrm{Si}$ and $\mathrm{Zr}$ ) and for which any correlations, if real, would have considerable impact on our understanding of stellar and/or globular cluster evolution. For all elements, the calculations indicate that adopting model atmospheres with appropriate compositions would result in only small changes to the derived abundances. For the correlations that we are investigating, the N-rich stars tend to have higher abundances $[\mathrm{X} / \mathrm{Fe}]$ than their $\mathrm{N}$-poor counterparts. That is, the calculations indicate that the correlations between $[\mathrm{X} / \mathrm{Fe}]$ and $[\mathrm{N} / \mathrm{Fe}]$ would in fact be strengthened had we adopted model atmospheres with appropriate compositions. Therefore, we tentatively rule out the possibility that the correlations between $\mathrm{N}$ and heavier elements are due to using model atmospheres with scaled-solar compositions. Nevertheless, a more detailed analysis using model atmospheres with the appropriate, but presently unknown, abundances of $\mathrm{He}$ and $\mathrm{C}$ would be of great interest.

As far as we are aware, correlations between the abundances of light elements ( $\mathrm{N}$ and $\mathrm{Al}$ ) and elements heavier than Si have not been noted in any other globular clusters. We attribute our detection primarily to the highly accurate and homogeneous stellar parameters derived from the Grundahl et al. (1999) Strömgren photometry, as well as to the high-quality spectra and large sample size.

\subsection{Implications for Globular Cluster and Stellar Evolution}

Having demonstrated that the Strömgren $c_{1}$ index (or $c y$ index) directly traces the $\mathrm{N}$ abundance, we confirm and reiterate the claim by Grundahl et al. (2000) that every globular cluster exhibits a considerable star-to-star variation in $\mathrm{N}$ abundances at all evolutionary stages as seen via the $c_{1}$ index. In Figure 14 we plot $V$ versus $c y$ for a subset of the Grundahl et al. (1999) clusters. Every cluster displays a dispersion in $c y$ comparable to that seen in NGC 6752 , indicating that all globular clusters exhibit a $\simeq 2.0$ dex dispersion in $[\mathrm{N} / \mathrm{Fe}]$, the value found in NGC 6752. This figure clearly shows that all clusters exhibit a large dispersion in $c y$, i.e., $\mathrm{N}$ abundances, at all evolutionary phases. Note that these clusters cover a range in metallicities: $[\mathrm{Fe} / \mathrm{H}] \simeq-1.2(\mathrm{NGC} 288, \mathrm{NGC}$ $362, \mathrm{M} 5),[\mathrm{Fe} / \mathrm{H}] \simeq-1.6(\mathrm{M} 3, \mathrm{M} 13, \mathrm{NGC} 6752)$, and $[\mathrm{Fe} / \mathrm{H}] \simeq$ -2.0 (NGC 6397, M15, M92).

Pollution by ejecta from intermediate-mass AGB stars and/or massive stars is the currently favored explanation for the star-tostar abundance variation of light elements. Both scenarios are problematic, with issues relating to the initial mass function and the predicted nucleosynthesis yields (Fenner et al. 2004; Decressin et al. 2007).

The handful of clusters for which $\mathrm{N}$ abundances have been measured in large samples of stars show a variety of $\mathrm{N}$ abundance distributions. M71 and 47 Tuc appear bimodal, whereas M5, M13, and M15 do not appear bimodal (Cohen et al. 2005 and references therein). As discussed above, the $[\mathrm{N} / \mathrm{Fe}]$ distribution for NGC 6752, using the relation between $\mathrm{N}$ and Strömgren photometry, appears to increase toward increasing [N/Fe]. Such a distribution is similar to that seen in the comparably metal-poor cluster M13 (and possibly also in M5). Curiously, both NGC 6752 and M13 exhibit bimodal CN distributions, as do all clusters in which CN can be measured (Smith 1987). The varying $[\mathrm{N} / \mathrm{Fe}]$ distributions in clusters of different metallicities will provide important observational constraints on the origin of the abundance anomalies in future globular cluster chemical evolution studies similar to that conducted by Fenner et al. (2004).

The correlations between $\mathrm{N}$ and heavy elements presented in this paper offer support for contributions from both AGB and massive stars to the observed abundance anomalies. Although quantitative yields of $s$-process elements from intermediate-mass metal-poor AGB stars are rare, $s$-process elements are expected to be synthesized, to some degree, in these stars (Busso et al. 2001). Fe-peak and $\alpha$-elements are synthesized by massive stars, at all metallicities (Chieffi \& Limongi 2004). Given that not every 


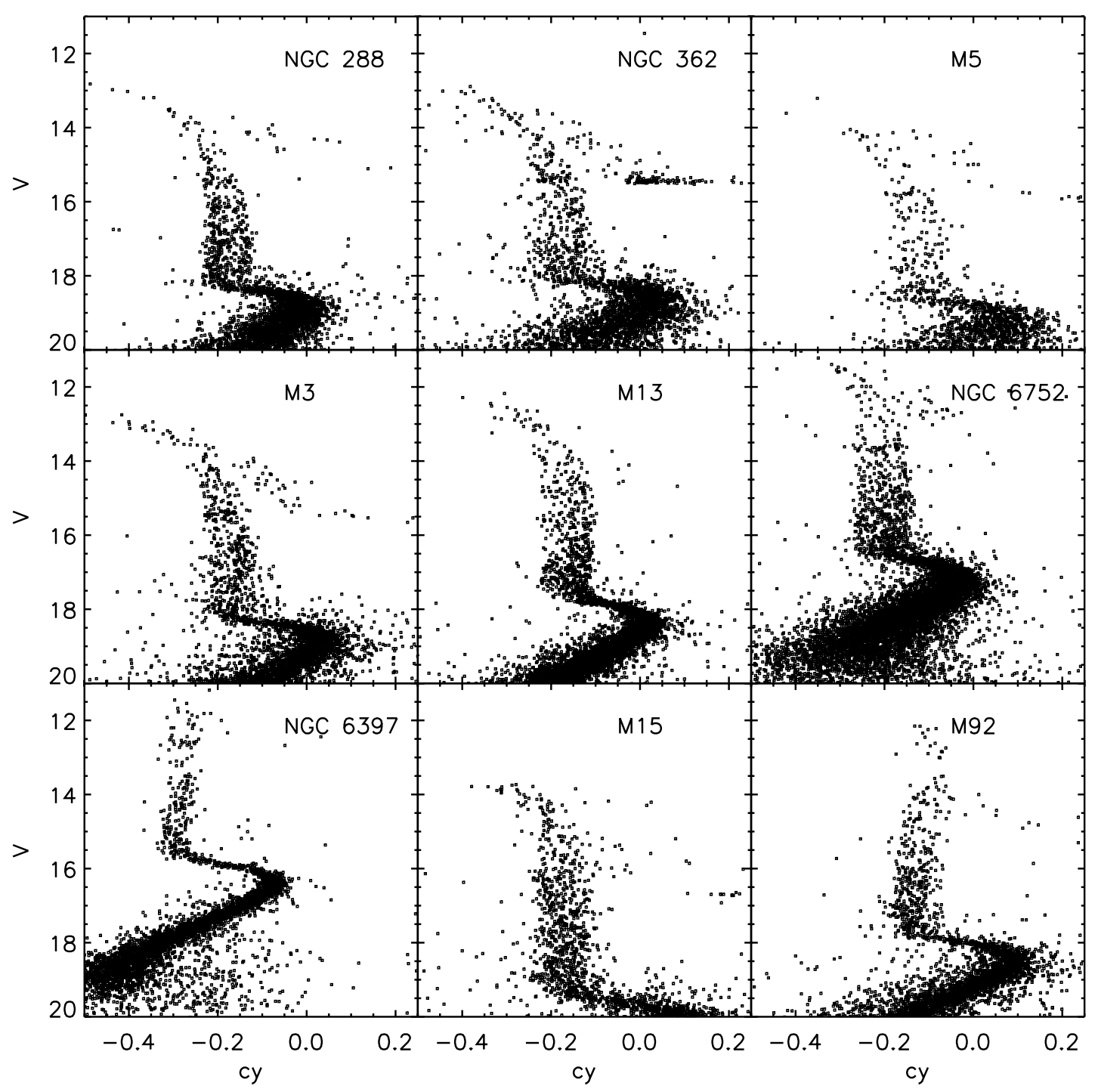

FIG. 14.-The $V$ vs. $c y$ color-magnitude diagrams for a sample of clusters taken from Grundahl et al. (1999). All clusters exhibit a large range in $c y$ at all evolutionary stages.

element is correlated with $\mathrm{N}$ at a statistically significant level, and that the amplitude of the heavy-element abundance variation is small, our results do not offer definitive support for either the massive star or AGB pollution scenarios.

As mentioned, target selection via the Strömgren $c_{1}$ index has allowed us to sample the full range of the star-to-star abundance variations, at this luminosity. Of great interest would be a further study of the full range of the abundance variations at different luminosities. An analysis of the most N-poor and N-rich stars, as selected via the $c_{1}$ index, would truly gauge any variation in the abundance anomalies as a function of evolutionary status. That the abundance variations for $\mathrm{O}-\mathrm{Al}$ have been found in unevolved stars suggests that primordial pollution processes rather than internal evolutionary processes must be the dominant mechanism, but these observations do not entirely preclude an internal evolutionary mechanism being a second-order effect. Indeed, the brightest stars in M13 appear to exhibit a systematic abundance variation for $\mathrm{O}, \mathrm{Na}, \mathrm{Mg}$, and Al with luminosity (Sneden et al. 2004). Such trends, if observed in additional clusters, would present new challenges for stellar evolution and stellar nucleosynthesis.

The Grundahl et al. (1999) Strömgren photometry extends to additional globular clusters. It would be of great interest to obtain high-resolution, high-S/N spectra for a large sample of stars within a narrow luminosity range in other globular clusters in order to see whether the correlations between light and heavy elements are also present. The key is to again use carefully calibrated Strömgren photometry to obtain homogeneous stellar parameters, such as in Grundahl et al. (2002).

Star-to-star helium abundance variations are expected when the $\mathrm{C}$-Al abundance anomalies are synthesized during hydrogen burning. NGC 2808 (D'Antona \& Caloi 2004) and $\omega$ Cen (Norris 2004) show evidence for He variations, based primarily on the interpretation of color-magnitude diagrams. Stellar models and isochrones computed by Salaris et al. (2006) show that the location of the RGB bump (weakly) depends on the He abundance. Recently, Carretta et al. (2007) found new evidence for He variations in NGC 6218 (M12) based on RGB stars. They looked at the luminosity functions for a sample of Na-rich and a sample of Na-poor stars, and found that the RGB bump differed by 0.05 mag, corresponding to a difference in $Y$ of 0.05 between the Na-poor and Na-rich populations. Although the correlation is not significant, we find marginal evidence for an increase in $[\mathrm{Fe} / \mathrm{H}]$ with increasing $[\mathrm{N} / \mathrm{Fe}]$. Such a correlation may be the signature of possible He variations.

Finally, one of the most stringent observational constraints on the origin of the abundance anomalies comes from $\mathrm{C}+\mathrm{N}+\mathrm{O}$, 
which is constant to within a factor of 2 (Smith et al. 2005). Predicted yields from AGB models are unable to satisfy this constraint (Lattanzio \& Tout 2006; Karakas et al. 2006). The ideal check of the constancy of $\mathrm{C}+\mathrm{N}+\mathrm{O}$ would be to measure $\mathrm{C}$ from $\mathrm{CH}, \mathrm{N}$ from $\mathrm{NH}$, and $\mathrm{O}$ from [O I] using high-resolution spectra for a sample that covers the full range of the abundance variations. We have measured two of these three indicators, but unfortunately our spectra do not cover the $4300 \AA \mathrm{CH}$ molecular lines. Measurement of $\mathrm{C}$ from $\mathrm{CH}$ for a subset of the most $\mathrm{N}$-rich and $\mathrm{N}$-poor stars in this sample is highly desired to examine the constancy of $\mathrm{C}+\mathrm{N}+\mathrm{O}$, and to search for correlations between $\mathrm{C}+\mathrm{N}+\mathrm{O}$ versus $\mathrm{N}$.

\section{CONCLUDING REMARKS}

In this paper we present measurements of $\mathrm{N}$ abundances in 21 bright giant stars near the RGB bump of the globular cluster NGC 6752. The sample was chosen to span the full range of the Strömgren $c_{1}$ index at this luminosity. The amplitude of the $\mathrm{N}$ abundance variation at the sample's luminosity is 1.95 dex. We confirm that the $\mathrm{N}$ abundances are correlated with the $c_{1}$ index. We find a linear relation between the $\mathrm{N}$ abundances and a new index, $c y=c_{1}-(b-y)$. We apply this new relation to large numbers of RGB stars and find that the [N/Fe] distribution increases toward higher values. Broader implications are that all globular clusters show large star-to-star variations in their $c y$ indices (i.e., $\mathrm{N}$ abundances) at all evolutionary stages.

We find that the $\mathrm{N}$ abundances are correlated with the light elements $\mathrm{O}, \mathrm{Na}, \mathrm{Mg}$, and $\mathrm{Al}$, a feature that is seen in every wellstudied globular cluster. However, we find for the first time that the $\mathrm{N}$ abundances are also correlated with the abundances of $\mathrm{Si}$ and heavier elements. While such correlations are statistically significant, the amplitude of the variation is small $(<0.2$ dex variation in $[\mathrm{X} / \mathrm{Fe}]$ as $[\mathrm{N} / \mathrm{Fe}]$ varies by 2 dex). We attribute the detection to the large sample size, high-quality data, and most importantly to the homogeneous and precise stellar parameters obtained from the Grundahl et al. (1999) Strömgren photometry.

Analysis using model atmospheres with the appropriate N, O, $\mathrm{Na}$, and $\mathrm{Al}$ abundances gives very similar abundances to those derived using model atmospheres with scaled-solar compositions. Therefore, the correlations between $\mathrm{N}$ and heavier elements are unlikely to be due to using models with scaled-solar compositions. In fact, for the subset of elements investigated ( $\mathrm{Si}, \mathrm{Y}$, and $\mathrm{Zr}$ ), the correlations with $\mathrm{N}$ would be even more significant had we used models with appropriate compositions.

Of great interest would be to search for such correlations in an additional sample of stars at a different luminosity within this cluster and in other clusters. If correlations between light and heavy elements are again identified, they would offer support to both the AGB and massive star pollution scenarios for explaining the star-to-star abundance variation of light elements.

This research has made use of the SIMBAD database, operated at CDS, Strasbourg, France, and NASA's Astrophysics Data System. D. Y. thanks Mike Bessell, Amanda Karakas, and John Norris for helpful discussions. We thank the anonymous referee for helpful comments. This research was supported in part by NASA through the American Astronomical Society's Small Research Grant Program.
Alonso, A., Arribas, S., \& Martínez-Roger, C. 1999, A\&AS, 140, 261

Asplund, M., Gustafsson, B., Kiselman, D., \& Eriksson, K. 1997, A\&A, 318, 521

Briley, M. M., \& Cohen, J. G. 2001, AJ, 122, 242

Briley, M. M., Cohen, J. G., \& Stetson, P. B. 2002, ApJ, 579, L17

Briley, M. M., Harbeck, D., Smith, G. H., \& Grebel, E. K. 2004, AJ, 127, 1588

Briley, M. M., \& Smith, G. H. 1993, PASP, 105, 1260

Briley, M. M., Smith, V. V., Suntzeff, N. B., Lambert, D. L., Bell, R. A., \& Hesser, J. E. 1996, Nature, 383, 604

Buonanno, R., Caloi, V., Castellani, V., Corsi, C., Fusi Pecci, F., \& Gratton, R. 1986, A\&AS, 66, 79

Busso, M., Gallino, R., Lambert, D. L., Travaglio, C., \& Smith, V. V. 2001, ApJ, 557, 802

Carretta, E., Gratton, R. G., Lucatello, S., Bragaglia, A., \& Bonifacio, P. 2005, A\&A, 433, 597

Carretta, E., et al. 2007, A\&A, 464, 939

Chieffi, A., \& Limongi, M. 2004, ApJ, 608, 405

Cohen, J. G., Briley, M. M., \& Stetson, P. B. 2002, AJ, 123, 2525 2005, AJ, 130, 1177

Cohen, J. G., \& Meléndez, J. 2005, AJ, 129, 303

Cottrell, P. L., \& Da Costa, G. S. 1981, ApJ, 245, L79

Da Costa, G. S., \& Cottrell, P. L. 1980, ApJ, 236, L83

D’Antona, F., \& Caloi, V. 2004, ApJ, 611, 871

Decressin, T., Meynet, G., Charbonnel, C., Prantzos, N., \& Ekström, S. 2007, A\&A, 464, 1029

Denissenkov, P. A., Da Costa, G. S., Norris, J. E., \& Weiss, A. 1998, A\&A, 333, 926

D’Odorico, S., Cristiani, S., Dekker, H., Hill, V., Kaufer, A., Kim, T., \& Primas, F. 2000, Proc. SPIE, 4005, 121

Fenner, Y., Campbell, S., Karakas, A. I., Lattanzio, J. C., \& Gibson, B. K. 2004, MNRAS, 353, 789

Fulbright, J. P., McWilliam, A., \& Rich, R. M. 2007, ApJ, 661, 1152

Gratton, R., Sneden, C., \& Carretta, E. 2004, ARA\&A, 42, 385

Gratton, R. G., Sneden, C., Carretta, E., \& Bragaglia, A. 2000, A\&A, 354, 169

Gratton, R. G., et al. 2001, A\&A, 369, 87

Grevesse, N., Asplund, M., \& Sauval, A. J. 2007, Space Sci. Rev., 130, 105

Grevesse, N., \& Sauval, A. J. 1998, Space Sci. Rev., 85, 161

Grundahl, F., Briley, M., Nissen, P. E., \& Feltzing, S. 2002, A\&A, 385, L14

Grundahl, F., Catelan, M., Landsman, W. B., Stetson, P. B., \& Andersen, M. I. 1999, ApJ, 524, 242
Grundahl, F., Vandenberg, D. A., Stetson, P. B., Andersen, M. I., \& Briley, M. 2000, in The Galactic Halo: From Globular Cluster to Field Stars, ed. A. Noels et al. (Liège: Inst. d'Astrophys. et Geophys.), 503

Gustafsson, B., Bell, R. A., Eriksson, K., \& Nordlund, A. 1975, A\&A, 42, 407

Johnson, J. A., Herwig, F., Beers, T. C., \& Christlieb, N. 2007, ApJ, 658, 1203

Johnson, J. A., Ivans, I. I., \& Stetson, P. B. 2006, ApJ, 640, 801

Karakas, A. I., Fenner, Y., Sills, A., Campbell, S. W., \& Lattanzio, J. C. 2006, ApJ, 652, 1240

Karakas, A. I., \& Lattanzio, J. C. 2003, Publ. Astron. Soc. Australia, 20, 279

Kraft, R. P. 1994, PASP, 106, 553

Kurucz, R. 1993, CD-ROM 13, ATLAS9 Stellar Atmosphere Programs and 2 km/s Grid (Cambridge: SAO), 13

Langer, G. E., \& Hoffman, R. D. 1995, PASP, 107, 1177

Lattanzio, J. C., \& Tout, C. A. 2006, in Stars and Nuclei: A Tribute to Manuel Forestini, ed. T. Montmerle \& C. Kahane (Les Ulis: EDP), 189

Letarte, B., Hill, V., Jablonka, P., Tolstoy, E., François, P., \& Meylan, G. 2006, A\&A, 453, 547

Norris, J., Cottrell, P. L., Freeman, K. C., \& Da Costa, G. S. 1981, ApJ, 244, 205

Norris, J., \& Freeman, K. C. 1979, ApJ, 230, L179

Norris, J. E. 2004, ApJ, 612, L25

Osborn, W. 1971, Observatory, 91, 223

Popper, D. M. 1947, ApJ, 105, 204

Prantzos, N., \& Charbonnel, C. 2006, A\&A, 458, 135

Salaris, M., Weiss, A., Ferguson, J. W., \& Fusilier, D. J. 2006, ApJ, 645, 1131

Smith, G. H. 1987, PASP, 99, 67 2006, PASP, 118, 1225

Smith, V. V., Cunha, K., Ivans, I. I., Lattanzio, J. C., Campbell, S., \& Hinkle, K. H. 2005, ApJ, 633, 392

Sneden, C. 1973, ApJ, 184, 839

Sneden, C., Kraft, R. P., Guhathakurta, P., Peterson, R. C., \& Fulbright, J. P. 2004, AJ, 127, 2162

Suntzeff, N. B., \& Smith, V. V. 1991, ApJ, 381, 160

Ventura, P., \& D'Antona, F. 2005, ApJ, 635, L149

Yong, D., Grundahl, F., Lambert, D. L., Nissen, P. E., \& Shetrone, M. D. 2003 , A\&A, 402, 985

Yong, D., Grundahl, F., Nissen, P. E., Jensen, H. R., \& Lambert, D. L. 2005, A\&A, 438, 875

Zinn, R. 1977, ApJ, 218, 96 